\title{
Space optics: challenge and opportunity
}

\section{James B. Breckinridge}

James B. Breckinridge, "Space optics: challenge and opportunity," Proc. SPIE 5524, Novel Optical Systems Design and Optimization VII, (22 October 2004); doi: 10.1117/12.560474

SDIE Event: Optical Science and Technology, the SPIE 49th Annual Meeting, 2004, Denver, Colorado, United States 


\title{
Space Optics: Challenge and Opportunity
}

\author{
James B. Breckinridge \\ Jet Propulsion Laboratory \\ Pasadena, CA. 91109
}

\begin{abstract}
Innovative optical designs are needed to create the space sensor systems of the future. The NASA mission development process has created several very challenging design and engineering problems. Three of these are discussed: The SAFIR is a 15 to 25 meter clear aperture telescope cooled to 4 degrees Kelvin, with spectrographs and imaging systems cooled to 1 degree Kelvin. The Terrestrial Planet Finder (TPF) will detect and characterize planets in orbit about other stars, The Stellar Interferometer (SI) will image across the surfaces of distant stars. Issues related to optical design \& engineering and image quality will be discussed. This paper reviews the optical systems and engineering needs for next generation astrophysics missions.
\end{abstract}

\section{INTRODUCTION}

The combined scientific content of these two Themes address three compelling questions:

- What is the nature of the matter and energy that dominate the Universe?

- How does the Origin of stars and planets couple to the fundamental properties of space and time, energy and matter?

How did our Universe enable the formation of Life?

The NASA Astronomical Search for Origins Program, often-abbreviated "Origins" consists of 4 missions to detect and characterize life on planets outside our solar system. Each one significantly more complex than the previous and each one requiring incredible new technology - inventions and processes that stretch the imagination. These are the James Webb Space Telescope, the Space Interferometer Mission, Terrestrial Planet Finder, and Life Finder. Origins missions use new windows of high spatial resolution and hyper contrast optical systems to image astronomical objects at micro-arc second resolution and with extremely low backgrounds. One of the mission objectives of the origins program is to detect and characterize exo-solar system planets. Control within the optical system of unwanted radiation at the level of one part in 10 to the $12^{\text {th }}$ will be required ${ }^{1}$.

Large innovative space telescopes and instruments will also be needed for the Single Aperture FarInfrared Observatory, where a 10-meter aperture cooled to 4-degrees Kelvin and the Stellar Imager system where Astronomers will image the details across the surface of planets, using a large number of 8 to 10 meter telescopes formation flying to form an interferometer whose aperture is several tens of KM.

\section{TECHNOLOGY AREAS}

There are five broad areas into which these key-enabling capabilities can be grouped. These are:

- Detectors - Detectors are the transducers that convert the natural electromagnetic carrier of scientific information into electrical signals that can be measured and processed.

- Optics - Optics collect, concentrate, manipulate, and analyze electromagnetic radiation and includes the surfaces, precision substrates, and any associated active surface shape controls and wavefront sensors. 
- Cryogenic Thermal Control - For astrophysics, a key benefit of space is the ability to minimize the effects of thermal noise by reducing the background radiation of the instrument well below what is being measured. Cryogenic cooling of detectors and large optical surfaces is required to achieve this benefit.

- Precision Physical and Virtual Structures - The elements of physical, virtual, passive or active structure systems that maintain the precise alignment of optics, detectors and proof masses order to work as optical instruments and gravitational observatories. Wavefront sensing and control is required to create an optical structure (virtual or real) for very large space telescope systems, which must be of low mass for launch.

- Distributed Spacecraft Systems - Beyond the size limit of structurally connected systems, distributed spacecraft systems appear to be the means to fully exploit space for extremely high spatial resolution dilute or sparse aperture optical systems. Examples of needed capabilities include precision spacecraft attitude control system components, and micro-thrusters, hyper-precision dragfree capabilities, and efficient multi-spacecraft maneuver algorithms.

\section{Vision Missions}

Several vision mission studies by the scientific community are underway currently under support from the Science Mission Directorate at NASA. The information given below will charge as the science and technology teams continue their studies, however the need for the basic technologies probably will not change. Also the priority or sequence of these missions will change as the priorities of the Agency changes. The discussion below provides narrative description of enabling technologies for the purpose of illustrating the opportunities.

\section{SAFIR (Single Aperture Far-Infrared Observatory} SAFIR.

Dr. Dan Lester, of the University of Texas is leading the team the Mission Vision study of the

The Single Aperture Far-Infrared Observatory (SAFIR) will combine a large primary mirror with active cooling to provide unprecedented sensitivity at far-IR and submillimeter wavelengths. The observatory will study the formation of structures, from the first stars and galaxies in the distant universe to planetary systems around nearby stars.

\section{The science goals of this proposed mission are:}

1. To probe the epoch of re-ionization due to the first stars when the universe was less than $1 / 20$ its present age.

2. Trace the formation and evolution of star forming and active galaxies since their inception.

3. Explore the connection between black holes and their host galaxies.

4. Reveal the details of star and planet formation in nearby debris-disk systems.

5. Search for and quantify pre-biotic molecules in the interstellar medium.

\section{The required observatory characteristics are:}

1. Primary Mirror Diameter: $10 \mathrm{~m}$

2. Telescope Temperature: $5 \mathrm{~K}$ or lower

3. Wavelength Coverage: 20 microns to $1 \mathrm{~mm}$

\section{The instruments that the astronomers require on board are:}

1. Background-limited detector arrays with thousands of pixels for broadband imaging over the full wavelength range. 
2. Moderate resolution $(\mathrm{R} \sim 1000)$ spectrometers with background-limited sensitivity and near-unit fractional bandwidth.

3. Heterodyne spectrometers tunable over the full wavelength regime with quantum-noise limited performance.

\section{New technologies in detectors, optics, thermal and structures will be needed to enable this telescope system.}

1. Detectors:
a. Background limited direct detectors for continuum observations from 20-1000 um - 10,000 pixel focal plane array with single pixel sensitivity of NEP $1 \mathrm{e}-18 \mathrm{~W} / \mathrm{Hz}^{1 / 2}$
b. Background limited direct detectors for moderate $(\mathrm{R}<1000)$ resolution spectroscopy $-1,000$ pixel focal plane array with single pixel sensitivity of NEP $1 \mathrm{e}-20 \mathrm{~W} / \mathrm{Hz}^{1 / 2}$
c. Heterodyne receivers for high resolution spectroscopy (R up to 100,000) - single channel performance near quantum limit for $v$ up to $3 \mathrm{THz}$

2. Optics:

a. $10 \mathrm{~m}$ telescope for FIR/submm diffraction limited at 30um and shields to achieve BLIP

3. Thermal:

b. $4 \mathrm{~K}$ temperature for optics

a. Thermal management system to control temperature of optics (4K temperature with $1 \mathrm{~K}$ spatial uniformity over aperture and temperature stability of $0.1 \mathrm{~K}$ over TBD time), heat shields and other temperature critical components

b. Cryocooler for optics (4K, heat load?) and detector (100mK, heat load?)

4. Structures:

a. Structures stability and accuracy to support diffraction limited performance requirement and operational temperature

b. Deployable in single launch

Orbit:

Sun-Earth L2 point, (Alternatives, e.g., a 3 AU orbit, are under consideration.)

A successful SAFIR mission will require very innovative technology for success.

\section{LARGE APERTURE UV OPTICAL OBSERVATORY}

Dr. James Green of the University of Colorado is leading a team of scientists and technologists studying this mission.

The Large Aperture UV Optical Observatory is a 4 to 8 meter diffraction-limited optical system with imaging, spectral and polarization measurement capability across the ultraviolet region of the spectrum to observe large-scale structure of the universe, dark matter, baryons, the origin and evolution of the formation of the elements, galaxies and quasars. The Hubble Space Telescope science shows a wealth of information critically important to our understanding of the Origins of man in the Universe in the high-spatial resolution ultra-violet region of the spectrum. The JWST will not record data in a very important spatial-spectral region of the Universe below 0.6um, and therefore this telescope will full fill an important cornerstone to our understanding answers to the questions: Are we alone? How did we get here?

\section{Science Goals:}

1. Understand how stars and galaxies were formed out of the primordial matter in the universe

2. Understand the evolution of young Galaxies and the distribution of the elements 
3. Large scale structure and dark matter

4. Protostars and stellar jets

\section{Observatory Characteristics:}

1. Primary Mirror Diameter: 4 to 8 meters

2. Optics precision fabricated and coatings optimized for ultra-violet

3. Wavelength Coverage: 0.1 to 1.0 micrometers

\section{Instruments on board are under planning \& development}

1. High resolution imaging spectrometer $(\mathrm{R} \sim 10,000)$

2. Polarization analysis with a medium resolution $(\mathrm{R} \sim 1000)$ imaging spectrometer

3. Ultra-low light-level wide-field imager

4. Coronagraph, baffles, masks

\section{Enabling Capabilities are under planning \& development:}

1. Detectors:

a. $>10 \mathrm{Kx} 10 \mathrm{~K}$ solar blind photon counting array over $0.1-1 \mathrm{um}$

b. Photon counting and energy selective detectors with $\mathrm{R} \sim 100$

2. Optical System:

a. 4 to 8 meter diffraction limited at 0.1 micron super smooth mirror

b. Wavefront sensing and control

c. Control unwanted thermal radiation

d. Special purpose optical coatings

3. Thermal:

a. Cryocooler needed for photon counting and energy selective detectors

4. Structures:

a. Structures stability and accuracy to support diffraction limited performance requirement at the operational temperature

b. Robotically deployed, from a single launch, and autonomously aligned and controlled to initialize and sustain diffraction limited performance at 0.1 -micron wavelength.

c. $<.1$ milli arc second control and pointing

\section{Orbit:}

1. Sun-Earth L2 point, or Sun-Jupiter L2 point

\section{STELLAR INTERFEROMETER}

Dr. Kenneth Carpenter of the NASA Goddard Space Flight Center is leading this effort to define a mission vision for an interferometer system, which will provide information to reconstruct images across the surfaces of stars. The goal is to understand the various effects of magnetic field of stars, the dynamos that generate them, and the internal structure and dynamics of stars other than our sun.

\section{OTHER OPTICS CHALLENGES}

Other large space telescope systems in the preliminary phase at NASA include: Planet Imager, Life Finder, and The Far-Infrared and Submillimeter Interferometer.

\section{Conclusions:}

Astrophysics of this century will be defined by a series of large UV, Optical, Infrared and submillimeter space telescopes, probing ever deeper into the cosmos. Exciting technical challenges and opportunities are waiting for us. 
This paper reviewed the work of many scientists and engineers within the context of optical sciences and engineering challenges of the future. It is always difficult to predict the future, but the future of space astronomy most assuredly rests with the innovative optics and technology that is not available today.

\section{Acknowledgments}

The author acknowledges helpful discussion with many of his colleagues at NASA HQ and at NASA centers to create the content of this overview and summary invited paper.

This research was carried out at the Jet Propulsion Laboratory, California Institute of Technology, under a contract with the National Aeronautics and Space Administration.

\section{REFERENCES}

1. Detector Needs for Long Wavelength Astrophysics, A Report by the Infrared, Submillimeter, and Millimeter Detector Working Group, Eric Young(Chair), University of Arizona, June 2002,. NASA sponsored study. ${ }^{1}$

2. Origins Science Roadmap, NASA

3. Beyond Einstein Science Roadmap, NASA

4. J. Mather \& M. Clampin, Editors, Optical, Infrared, and Millimeter Space Telescopes, SPIE Proceeding 5487, 220 papers. 